\title{
Coexistencia de desnutrición crónica infantil y sobrepeso de sus madres en escolares de un colegio estatal de Lima
}

\author{
The coexistence of child chronic malnutrition and overweight of their mothers in students of a state \\ school in Lima
}

Sixto Reyna G. ${ }^{1}$; María E. Villanueva E².

\begin{abstract}
Resumen
El presente estudio determinó la prevalencia de problemas nutricionales y la coexistencia de desnutrición crónica infantil junto con el sobrepeso de las madres, en un colegio estatal de Lima. Los problemas nutricionales en escolares fueron anemia ( $41,03 \%$ ), sobrepeso (26,72 \%), retraso en el crecimiento (5,34 \%) y obesidad (3,82 \%). En las madres el exceso de peso fue de 64,89 \% y la prevalencia de anemia de 51,39\%; asimismo, el 20,65\% presentó altos niveles de grasa corporal (superior a $35 \%$ ), pero el 4,35\% reportó muy altos niveles de grasa corporal (superior a $40 \%$ en mujeres con menos de 39 años y superior a $41 \%$ en mujeres con más de 39 años); en el 39,36 \% de los casos se observó obesidad abdominal. La coexistencia de sobrepeso materno y retraso en el crecimiento de los escolares correspondió al 3,19\% de la muestra, 20,21 \% para el exceso de peso entre escolares y sus madres y 21,67\% para la coexistencia de anemia materna e infantil (los escolares). La ingesta de calorías en las madres de familia fue de 1.907,21 kcal y 1.926,45 kcal en niños; el exceso de peso se relacionaría con escasa actividad física o la subestimación del consumo de alimentos y la alta prevalencia de anemia, con la escasa ingesta de hierro. El perímetro de cintura se asoció con el Índice de Masa Corporal (IMC) y la proporción de grasa corporal en niños y mujeres.
\end{abstract}

Palabras clave: transición; nutricional; sobrepeso;desnutrición.

Abstract
This study determined the prevalence of nutritional problems and the coexistence of child chronic malnutrition with the overweight of their mothers in a state school in Lima. The nutritional problems in students were anemia (41,03\%), overweight (26,72\%), stunting 5,34 \% and obesity 3,82\%. In overweight mothers overweight was $64,89 \%$ and anemia prevalence $51,39 \%$, also $20,65 \%$ had high levels of body fat (above $35 \%$ ) while $4,35 \%$ reported very high levels of body fat (above $40 \%$ in women with less than 39 years of age, and $41 \%$ in women aged 39 years or more), abdominal obesity was observed in $39,36 \%$ of the cases. The coexistence of maternal overweight and stunting in schoolchildren corresponded to 3,19\% of the sample; $20,21 \%$ for overweight among school children and their mothers and $21,67 \%$ for the coexistence of maternal and child anemia. The calories intake in the mothers was $1.907,21 \mathrm{kcal}$ and $1.926,45 \mathrm{kcal}$ in children, the excess weight would be related to low physical activity and / or underestimation of food consumption, and the high prevalence of anemia would be related to the low iron intake. The waist circumference was associated with BMI and body fat percentage.

Keywords: nutrition; transition; overweight; chronic malnutrition.

\section{Introducción}

América Latina atraviesa por un estado de transición nutricional dando lugar a la coexistencia de obesidad y desnutrición en la población a nivel nacional, comunitario y familiar (Garret y Ruel, 2005). En el Perú, el $48 \%$ de los escolares padece desnutrición crónica, la que se exterioriza con retraso en el crecimiento; el $97 \%$ de los menores que están afectados por la desnutrición estudian en colegios estatales (Ministerio de Educación/UNICEF/
FOCONDES/PMA, 1993). Por otra parte, la prevalencia de sobrepeso materno es de 47,8 \%, encontrándose coexistencia, en el mismo hogar, de sobrepeso materno y niños de 6 a 60 meses con déficit de talla $(9,6 \%)$, sólo superado por Bolivia y Guatemala (Garret y Ruel, 2005; Min. Educación/UNICEF/FOCONDES/PMA, 1933); además, en los niños con retraso en el crecimiento se observan también alteraciones metabólicas (Grillo et al., 2000). Esto puede ayudar a explicar el incremento de la

\footnotetext{
1 Mg. en Nutrición. Universidad Nacional Agraria La Molina, Lima, Perú.

2 Profesora Principal, Departamento de Nutrición. Universidad Nacional Agraria La Molina, Lima, Perú. Email: mvillanueva@lamolina.edu.pe
} 
prevalencia de la obesidad en adultos y adolescentes de baja estatura en países en vías de desarrollo (Hoffman et al., 2000).

Ya que la circunferencia de la cintura se correlaciona con el tejido adiposo intra abdominal (Hirschler et al., 2005) y la obesidad abdominal se asocia con alteraciones en el perfil lipídico, riesgos cardiovasculares y trastornos metabólicos de niños, adolescentes y adultos (Pajuelo et al., 2004), se recomienda medir la circunferencia de cintura en niños con un Índice de Masa Corporal (IMC) superior a 85 percentil (Piazza, 2005).

La deficiencia de hierro reduce la capacidad de trabajo de los individuos y las poblaciones, especialmente en los sectores más pobres y menos educados, causando serias consecuencias sociales y económicas. En el Perú, la mediana de consumo de hierro en escolares de 5 a 14 años es de 7,2 mg/día y la prevalencia de anemia $(\mathrm{Hb}<11,5 \mathrm{gr} /$ dl) es de $53,6 \%$; en mujeres en edad fértil la mediana de consumo de hierro es de $7,4 \mathrm{mg}$ /día y la prevalencia de anemia $(\mathrm{Hb}<12 \mathrm{mg} / \mathrm{dl})$ es de 38,2 \% (INS, 2008).

Se ha determinado la asociación entre el sobrepeso y la prevalencia de anemia en mujeres (Pajuelo et al., 2000) y niños (Pajuelo et al., 2001); al respecto, señalamos que la deficiencia de hierro es la causa más común de anemia en el mundo (Frewin et al., 1997); esta se presenta cuando la absorción de hierro es deficiente para cubrir los requerimientos del organismo y puede ser atribuida sea al bajo consumo de hierro como al incremento de sus necesidades, entre ellas la parasitosis, la menstruación y la gestación (Stoltzfus et al., 1997; Frewin et al., 1997). En niños, la deficiencia de hierro está asociada con el estado socioeconómico, la desnutrición crónica (Hassan et al., 1997) y el estatus de hierro en la madre (Perez et al., 2005). Ya que la obesidad, la desnutrición crónica y la anemia son problemas de salud pública que pueden coexistir en una misma persona y en el hogar, el objetivo de la presente investigación fue cuantificar la prevalencia de la coexistencia de desnutrición crónica infantil y sobrepeso de las madres, en un grupo de niños matriculados en un colegio estatal de Lima.

\section{Materiales y métodos}

La presente investigación se realizó en el distrito La Molina de Lima y los datos se obtuvieron del colegio estatal $\mathrm{N}^{\circ}$ 1140 “Aurelio Miró Quesada". El tamaño de la muestra se determinó considerando una prevalencia de sobrepeso y anemia del $50 \%$, un nivel de confianza del $95 \%$ y un error muestral del $10 \%$, lo que significó una muestra de 96 unidades de estudio. Para determinar el estado nutricional, se consideraron indicadores antropométricos aplicados a 131 niños y 94 mujeres; la concentración de hemoglobina fue evaluada en 117 niños y 72 madres de familia los cuales consintieron en participar en este estudio.

Tanto en los niños como en sus madres, se determinó peso, estatura, circunferencia de cintura y proporción de grasa corporal; a la vez, se estableció la concentración de hemoglobina y se estimó la ingesta de macronutrientes (carbohidratos, lípidos y proteínas), hierro y distribución energética de la dieta.
El peso corporal se valoró empleando una balanza digital marca Miray, modelo BMD-23. Los participantes fueron pesados con ropa ligera y descalzos, registrando el peso completo en $\mathrm{kg}$ con precisión de 100 gramos; la estatura fue descrita en centímetros, se utilizó un tallímetro portátil con una precisión de un milímetro; los datos correspondientes a la circunferencia de cintura se obtuvieron empleando una cinta métrica inextensible y midiendo la circunferencia de la cintura entre el arco costal y la cresta iliaca.

La concentración de hemoglobina se estableció empleando el método de cianometahemoglobina, usando un espectrofotómetro marca Perkin Elmer, modelo Lambda EZ 150 y hemogloWiener reactivo y Estándar, Wiener lab ${ }^{\circledR}$. En escolares, el punto de corte fue de $11,5 \mathrm{mg}$ hemoglobina/dl y en mujeres de 12,0 mg hemoglobina/dl (WHO, 2001); la proporción de grasa corporal se estimó por bioimpedancia empleando la balanza digital ya citada, los resultados de los porcentajes de grasa corporal se interpretaron según los criterios expuestos por Gallagher et al., 2000.

La ingesta calórica y de macronutrientes de los participantes se calculó mediante un recordatorio de 24 horas empleando un laminario de medidas caseras (PRISMA, 1996). Se efectuaron dos recordatorios para cada miembro de la unidad de estudio (pareja madreniño) en el curso de una semana. La sub muestra que se empleó para recabar la información de ingesta calórica y de macronutrientes incluyó 14 unidades de estudio. La información se recabó en la institución educativa o en el domicilio de los participantes. Se calculó la composición calórica y de macronutrientes de cada alimento consumido a partir de la tabla peruana de composición de alimentos (Collazos, 2004); esta información se contrastó con los requerimientos de energía en niños que se expresó en kcal/ día (FAO/OMS/UNU, 2001). En las mujeres se consideró como desnutrición un IMC inferior a $18,5 \mathrm{~kg} / \mathrm{m}^{2}$ y normal un IMC ubicado entre 18,5 y $25 \mathrm{~kg} / \mathrm{m}^{2}$; el sobrepeso se determinó con un IMC superior a 25 e inferior a $30 \mathrm{~kg}$ / $\mathrm{m}^{2}$ y la obesidad se estableció cuando el IMC fue mayor a $30 \mathrm{~kg} / \mathrm{m}^{2}$. Se consideró como obesidad abdominal cuando la circunferencia de cintura (CC) fue superior a $88 \mathrm{~cm}$ (American Medical Association, 2003).

El estado nutricional de los niños fue evaluado de acuerdo con el sexo y la edad de los participantes, empleando como indicadores el IMC y la talla para la edad establecidos por el Centers for Disease Control and Prevention -CDC(National Center for Health Statistics, 2000), definiéndose como límites de la normalidad dos desviaciones estándar de la mediana referencial.

Las variables se analizaron por medio de estadísticas descriptivas y se presentaron como promedios \pm una desviación estándar (DE) e intervalos de confianza (95\%) en el caso de variables continuas y como porcentajes en el caso de variables nominales. Se compararon medias entre el grupo de niños de acuerdo con su sexo mediante la prueba $\mathrm{t}$ de Student para variables continuas. Para establecer asociación entre variables relacionadas con el estado nutricional y entre este y la ingesta de macronutrientes, se emplearon análisis de regresión y correlación, igual 
procedimiento se empleó para establecer la asociación entre el nivel de hemoglobina y la ingesta de hierro. Se consideraron estadísticamente significativos los resultados con un nivel de significación igual o menor a 0,05. Para el análisis estadístico se empleó el paquete estadístico Minitab versión 14.

El estudio incluyó niños y niñas entre 7 y 11 años de edad, con autorización de los padres o tutores, que asistían a la escuela pública “Aurelio Miró Quesada” N 1140 del distrito La Molina en Lima. Las mujeres participantes se encontraban en edad fértil (15 a 49 años) y eran madres de un escolar integrante del estudio, los criterios de exclusión fueron: obesidad originada por patologías, alteración de la distribución de agua corporal en los niños por enfermedades, ingesta de diuréticos o esteroides. En ninguno de los incluidos hubo presencia de edemas ni afecciones generalizadas de la piel o con asimetrías corporales (amputaciones), también se excluyó niños que hubiesen presentado cualquier tipo de infección o proceso inflamatorio durante el mes anterior a su participación en el estudio, los mismos criterios fueron aplicados para las madres.

\section{Resultados y discusión}

La prevalencia de problemas nutricionales en escolares se observa en la Tabla 1, la circunferencia de cintura (CC) se asoció de manera directa con el IMC $(r=0,775, \mathrm{P}<0,001)$ y también de manera positiva con la talla de los escolares $(\mathrm{r}=0,321, \mathrm{P}<0,001)$.

La prevalencia de problemas nutricionales en madres de familia se detalla en la Tabla 2, el IMC se asoció de manera significativa con la circunferencia de cintura $(\mathrm{r}=0,866 ; \mathrm{P}<$ $0,001)$ y con el porcentaje de grasa corporal $(\mathrm{r}=0,661 ; \mathrm{P}<$ 0,001), de igual manera, el índice de correlación entre la CC

Tabla 1. Problemas nutricionales en escolares (niños y niñas)

\begin{tabular}{lccc}
\hline Problema nutricional & Prevalencia niños (\%) & Prevalencia niñas (\%) & Prevalencia total (\%) \\
\hline Anemia & 46,31 & 36,51 & 41,03 \\
Sobrepeso & 32,79 & 21,43 & 26,72 \\
Obesidad & 4,92 & 2,86 & 3,82 \\
Retraso crecimiento & 4,92 & 5,72 & 5,34 \\
\hline
\end{tabular}

Tabla 2. Prevalencia de problemas nutricionales en madres de familia

\begin{tabular}{lr}
\hline Problemas nutricionales & Prevalencia (\%) \\
\hline Sobrepeso & 47,87 \\
Obesidad & 17,02 \\
Obesidad abdominal & 39,36 \\
Anemia & 51,39 \\
Alta proporción de grasa corporal & 25 \\
\hline
\end{tabular}

y el porcentaje de grasa corporal es significativo $(r=0,585$, $\mathrm{P}<0,001)$; por otra parte la concentración de hemoglobina no se asoció de manera significativa con el porcentaje de grasa corporal $(\mathrm{r}=0,022 ; \mathrm{P}=0,855)$, circunferencia de cintura $(r=-0,005 ; p=0,964)$ ni IMC $(-0,014)$.

La coexistencia de sobrepeso materno y retraso en el crecimiento de escolares correspondió al 3,19\% de la muestra; el exceso de peso en escolares y sus madres fue del $20,21 \%$ de la muestra; la coexistencia de anemia en las madres y en los escolares afectaba al 21,67\%; el sobrepeso

Tabla 3. Consumo y distribución de energía en la dieta en escolares por grupo de edades

\begin{tabular}{|c|c|c|c|c|c|}
\hline Edad años & $\begin{array}{l}\text { Req. energía } \\
\text { (kcal) }\end{array}$ & $\begin{array}{c}\text { Consumo energía } \\
\text { (kcal) }\end{array}$ & $\begin{array}{l}\text { Proteínas (kcal) } \\
\text { Distribución } \\
\text { energética }(\%)\end{array}$ & $\begin{array}{l}\text { Lípidos kcal } \\
\text { Distribución } \\
\text { energética (\%) }\end{array}$ & $\begin{array}{l}\text { Glúcidos kcal } \\
\text { Distribución } \\
\text { energética }(\%)\end{array}$ \\
\hline 7 & $1.904,92$ & $1.569,9 \pm 317,8$ & $\begin{array}{l}229,71 \\
(14,42)\end{array}$ & $\begin{array}{l}422,07 \\
(26,5)\end{array}$ & $\begin{array}{l}941,00 \\
(59,08)\end{array}$ \\
\hline 8 & $2.148,24$ & $2.436,0 \pm 324,7$ & $\begin{array}{l}293,41 \\
(12,06)\end{array}$ & $\begin{array}{l}538,49 \\
(22,13)\end{array}$ & $\begin{array}{c}1.601,84 \\
(65,82)\end{array}$ \\
\hline 9 & $2.062,88$ & $2.004,8 \pm 651,7$ & $\begin{array}{l}343,29 \\
(16,93)\end{array}$ & $\begin{array}{l}492,65 \\
(24,30)\end{array}$ & $\begin{array}{c}1.191,34 \\
(58,77)\end{array}$ \\
\hline 10 & $2.520,97$ & $1.850,7 \pm 479,2$ & $\begin{array}{l}267,18 \\
(14,41)\end{array}$ & $\begin{array}{l}418,19 \\
(22,55)\end{array}$ & $\begin{array}{c}1.169,28 \\
(63,05)\end{array}$ \\
\hline 11 & $2.538,71$ & $1.711,3 \pm 157,2$ & $\begin{array}{l}213,53 \\
(12,39)\end{array}$ & $\begin{array}{l}560,57 \\
(32,52)\end{array}$ & $\begin{array}{l}949,73 \\
(55,09)\end{array}$ \\
\hline PROMEDIO & $2.235,14$ & $1.914,54$ & $\begin{array}{l}269,42 \\
(14,04)\end{array}$ & $\begin{array}{c}486,39 \\
(25,6)\end{array}$ & $\begin{array}{c}1.170,64 \\
(60,36)\end{array}$ \\
\hline
\end{tabular}


Tabla 4. Ingesta de nutrientes y distribución energética en madres de familia

\begin{tabular}{lcc}
\hline \multicolumn{1}{c}{ Ingesta Energía } & kcal & $\begin{array}{c}\text { Distribución } \\
\text { Energética (\%) }\end{array}$ \\
\hline Total & $1907,21 \pm 533,5$ \\
Proteínas & $240,62 \pm 74,4$ & 12,62 \\
Lípidos & $499,54 \pm 138,47$ & 26,19 \\
Carbohidratos & $1167 \pm 391,12$ & 61,19 \\
INGESTA DE HIERRO & \multicolumn{2}{c}{$8,12 \mathrm{mg} \mathrm{diarios}$} \\
CONCENTRACIÓN DE Hb & \multicolumn{2}{c}{$11,85 \mathrm{mg} / \mathrm{dl}$} \\
\hline
\end{tabular}

materno y la anemia en escolares correspondía al 41,46\%; la obesidad de las madres y la anemia en los escolares se daba en el $40 \%$ de la muestra.

La media de consumo de energía en escolares fue de $1.926,45 \mathrm{kcal}$, la energía proveniente de proteínas fluctuó entre $12,06 \%$ y $16,93 \%$; para los lípidos fue de $22,13 \%$ y $32,52 \%$ y los carbohidratos de $55,09 \%$ a $65,82 \%$; la media de consumo de hierro en escolares fue de $10,9 \mathrm{mg} /$ día, la concentración media de hemoglobina en escolares fue $11,53 \mathrm{mg} / \mathrm{dl}$, el consumo de energía y distribución energética en escolares se describe en la Tabla 3.

En las madres, la ingesta de energía promedio fue de $1.907,21 \mathrm{kcal}$; la energía alimentaria provino de las proteínas en un $12,62 \%$; de los lípidos en un $26,19 \%$ y de los carbohidratos en un $61,19 \%$. La ingesta media de hierro en las madres de familia fue de $8,12 \mathrm{mg} / \mathrm{dl}$ día y la concentración media de hemoglobina fue de $11,85 \mathrm{mg} / \mathrm{dl}$, estos resultados se aprecian en la Tabla 4.

\section{Discusión}

Se estableció que en el 3,19\% de la muestra coexistía exceso de peso materno y retraso en el crecimiento de sus hijos (los escolares objeto del estudio); el 20,21 \% presentó coexistencia de exceso de peso en los escolares y sus madres; mientras en el 21,67\% se observó coexistencia de anemia sea en las madres que en los escolares; la prevalencia de la coexistencia de sobrepeso materno/anemia en escolares y obesidad materna/anemia en escolares fue de $11,11 \%$ y $2,56 \%$, respectivamente.

\section{Estado nutricional de los escolares}

La prevalencia de problemas nutricionales en alumnos se aprecia en la Fig. 1. En el caso del sobrepeso este fue de $26,72 \%$, la obesidad de $3,82 \%$ y el retraso en el crecimiento del $5,34 \%$, mientras la prevalencia de la anemia se observó en el 41,03\%; las proporciones entre ambos sexos no fueron significativamente diferentes. La circunferencia de cintura (CC) se asoció de manera directa con el IMC de los escolares $(r=0,775, \mathrm{P}<0,001)$, la ecuación de regresión (b) circunferencia de cintura en escolares (niños y niñas) $=59,6+5,86$ IMC escolares, implica que el incremento de una unidad en el IMC de los escolares se traduce en el aumento de $5,86 \mathrm{~cm}$ en el perímetro de cintura, el coeficiente de determinación $\left(\mathrm{r}^{2}=\right.$ $0,600)$ permite atribuir el $60 \%$ del incremento de cintura al incremento del IMC.
La CC también se asocia de manera positiva con la talla de los escolares $(\mathrm{r}=0,321, \mathrm{P}<0,001)$, la ecuación de regresión (b): Circunferencia de cintura escolares (niños y niñas) $=$ $64,7+2,79 \mathrm{~T} / \mathrm{E}$ escolares indica que la CC se incrementó en $2,79 \mathrm{~cm}$ al incrementarse el indicador talla para la edad en una desviación estándar.

La prevalencia de obesidad se ha incrementado en las últimas décadas, tanto en adultos como en niños y se asocia con hipertrigliceridemia (Pajuelo, Rocca, Gamarra, 2003)

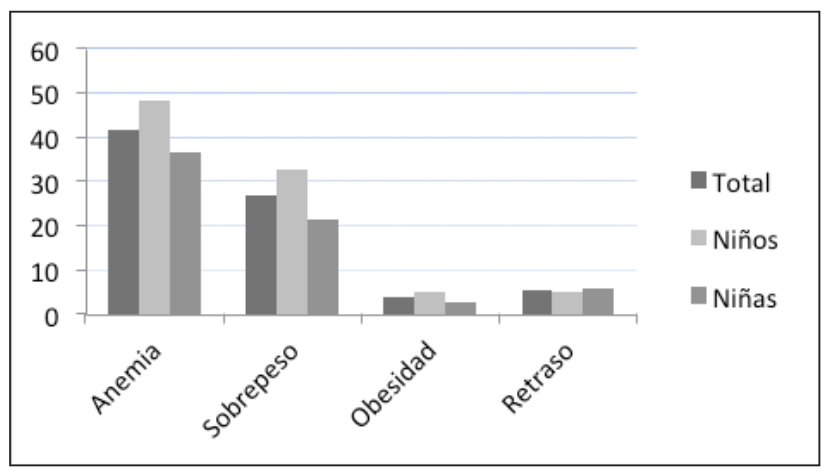

Figura 1. Prevalencia de problemas nutricionales en escolares

y con bajos niveles d HDLc, lo que sugiere un mayor riesgo de enfermedad cardiovascular porque el sobrepeso en niños y adolescentes es predictor de sobrepeso, morbilidad y mortalidad en la edad adulta (Guo y Cameron, 1999). Por otra parte, los niños desnutridos, con baja talla o con deficiencias de micronutrientes, tienen mayor riesgo de resultar en el futuro obesos, con mayor prevalencia de enfermedades crónicas no transmisibles y con menor capacidad intelectual y laboral, el retardo del crecimiento (desnutrición crónica) es particularmente importante debido a sus efectos negativos sobre el desarrollo de los individuos y la sociedad y es la manifestación más grave de la falta de acceso a los alimentos (CEPAL/FAO/PMA, 2008). En escolares limeños de 6 a 9 años, Pajuelo (2001) señala una prevalencia de sobrepeso de $14,1 \%$ y $10 \%$ de obesidad; con niños de 6 a 10 años, Llanos (2003) reportó una prevalencia de sobrepeso de 9,78 \% y 5,57\% de obesidad en varones y $9,78 \%$ de sobrepeso y $5,55 \%$ de obesidad en niñas. Torres, Caballero, Ponce y Valverde (2004) registraron $11,1 \%$ de desnutrición crónica, el 23,8 $\%$ de sobrepeso y el $23,2 \%$ de obesidad. 
La prevalencia de sobrepeso en el presente estudio (26,72 $\%$ ) es superior a las citadas, sin embargo la prevalencia de obesidad $(3,82 \%)$ es notoriamente inferior a la observada en los estudios mencionados; en la presente investigación la prevalencia de retraso en el crecimiento fue de 5,34\%, inferior al 11,11\% reportado por Torres et al., (2004).

En Perú se ha observado, en menores de tres años, una reducción de la prevalencia de retraso en el crecimiento de $54,1 \%$ en el año 2000 a 36,9\% en el 2004 (Lechtig et al., 2009); de la misma manera Gross et al. (2006) mencionan que en América Latina la reducción de la prevalencia de la desnutrición crónica es de aproximadamente del $1 \%$ anual, lo que podría explicar la baja prevalencia de retraso en el crecimiento encontrada en la presente investigación. Una prevalencia del retraso en el crecimiento inferior al $20 \%$ se considera que es de baja severidad (FAO, 2007). La circunferencia de cintura (CC) es un factor de riesgo importante para desarrollar el Síndrome Metabólico en la infancia, en el promedio de CC de los escolares de 7 a 11 años, evaluados en el presente estudio, se observa una tendencia a incrementarse de los 7 a los 10 años $(57,79 \mathrm{~cm}$ y $66,66 \mathrm{~cm}$, respectivamente) y a disminuir en escolares de 11 años a 62,42 cm. La prevalencia de anemia presenta amplios rangos, se estimó en $0,6 \%$ en escolares de Medellín-Colombia (Agudelo et al., 2003) y de 16,6 $\%$ en la Amazonía ecuatoriana (Quizphe et al., 2003). Pajuelo (2001) estableció una prevalencia de anemia de $69,3 \%$ en escolares de Matucana, Santa Eulalia y Lima, en el presente estudio la prevalencia de anemia en escolares fue de $41,03 \%$, se consideran como factores de riesgo de anemia la pobreza, la baja ingesta de hierro disponible y la pérdida de hierro (Queiroz et al., 2009).

\section{Problemas nutricionales en madres}

La prevalencia de sobrepeso y obesidad $(47,87 \%$ y $17,02 \%$, respectivamente) fue superior a los hallazgos de Miespireta, Rosas, Velásquez, Lescano y Lanata (2007), que reportaron el $34,4 \%$ de sobrepeso y el $12,7 \%$ de obesidad en mujeres; la obesidad abdominal, uno de los cuadros que configuran el Síndrome Metabólico, alcanzó el 39,36\%, en tanto la prevalencia de anemia fue del $51,39 \%$. La anemia se relaciona con una baja talla, lo que posteriormente podría traducirse en problemas en el parto, incrementando la mortalidad materna y neonatal; según la Organización Mundial de la Salud una prevalencia de anemia superior al $40 \%$ se considera como un severo problema de salud pública (WHO, 2001). En la Fig. 2 se

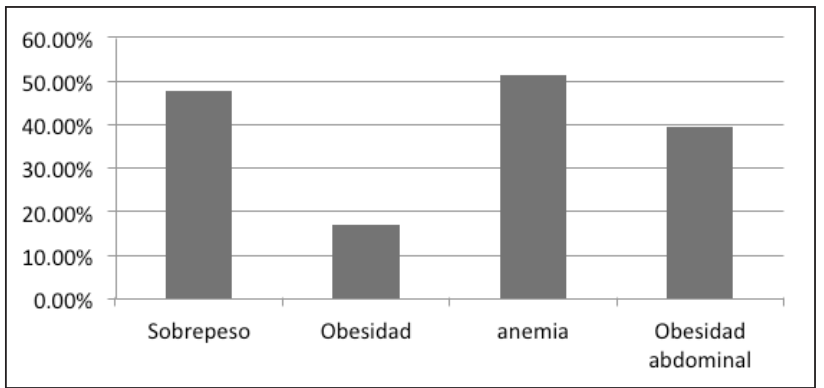

Figura 2. Problemas nutricionales en madres de familia aprecia la prevalencia de los problemas nutricionales en las madres hallados en la presente investigación.

El IMC se asoció de manera significativa con la CC $(\mathrm{r}=0,866 ; \mathrm{P}<0,001)$ y con el porcentaje de grasa corporal $(\mathrm{r}=0,661 ; \mathrm{P}<0,001)$; por otra parte, la concentración de hemoglobina no se asoció de manera significativa con el porcentaje de grasa corporal $(\mathrm{r}=0,022 ; \mathrm{P}=0,855)$, circunferencia de cintura $(r=-0,005 ; P=0,964)$ ni con IMC $(-0,014)$

Prevalencia de la coexistencia de problemas nutricionales en escolares y sus madres

En madres con sobrepeso, se reporta el 44,74\% de anemia en sus hijos, el índice de correlación entre el IMC materno y la concentración de hemoglobina en escolares fue muy bajo y negativo $(r=-0,069)$, siendo la asociación entre ambas variables prácticamente nula, el 40,74\% de los escolares hijos de madres no anémicas presentó anemia, mientras que el 39,39\% de las madres anémicas tenían hijos anémicos $(21,67 \%$ de la muestra), la concentración de hemoglobina fue ligeramente superior en madres que en sus hijos (11,76 mg/dl y 11,62 mg/dl, respectivamente), observándose un bajo grado de relación entre ambas variables; la circunferencia de cintura en mujeres con obesidad central $(94,39 \mathrm{~cm})$ fue significativamente superior $(\mathrm{p}<0,001)$ al perímetro de cintura de mujeres con una circunferencia de cintura inferior a $88 \mathrm{~cm}(80,61 \mathrm{~cm})$; sin embargo esto no fue evidente al comparar el perímetro de cintura de sus hijos $(\mathrm{p}=0,609)$. El perímetro de cintura en niños cuyas madres no presentaron obesidad central fue de $62,21 \mathrm{~cm}$ y de $62,97 \mathrm{~cm}$ en los hijos de mujeres con obesidad abdominal, la asociación entre los perímetros de cintura de los escolares y de sus madres fue baja y no significativa $(r=0,157, p=0,13)$ e inferior al registrado por Farías et al., 2007 ( $\mathrm{r}=0,365, \mathrm{p}<0,001)$.

\section{Ingesta de energía y nutrientes}

El consumo de energía no fue diferente entre los grupos evaluados $p=0,19$, estos resultados difieren de lo señalado por Wait, Blair y Roberts (1969), que observaron incremento sostenido de la ingesta de calorías hasta los 20 años en varones y los 13 años en mujeres. En el presente estudio la ingesta de energía no se asoció con el IMC ( $\mathrm{r}=$ $0,034, \mathrm{P}=0,905)$, esta asociación es mayor entre la ingesta de energía y el indicador talla para la edad. $(\mathrm{r}=0,485 ; \mathrm{P}=$ 0,069), lo que concuerda con lo manifestado por Wait, Blair y Roberts (1969), al relacionar las necesidades energéticas con la tasa de crecimiento antes que con la masa corporal. La media de consumo de energía por parte del grupo evaluado fue de $1.926,45 \mathrm{kcal}$, probablemente debido a que en la etapa de crecimiento el desarrollo de los tejidos óseo y muscular es relativamente mayor que la deposición de tejido adiposo (Sawaya y Roberts, 2003). Por otra parte, se observa que el perímetro de cintura no guarda relación $(\mathrm{r}=0,042 \mathrm{P}=0,881)$ con la ingesta de energía entre los escolares evaluados; en la Fig. 3 se observa la tendencia de consumo total de energía (expresado en kcal) y la energía proveniente de proteínas, lípidos y carbohidratos.

La distribución energética de estas dietas fue similar a las recomendaciones dietéticas del Deparment of Health 


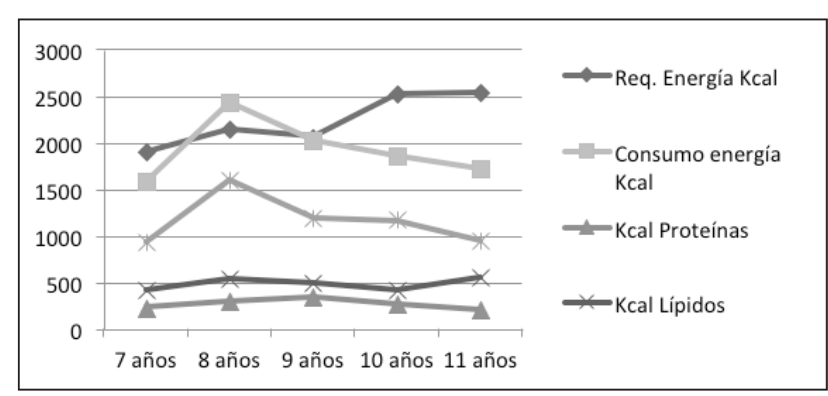

Figura 3. Requerimientos y consumo de energía en escolares

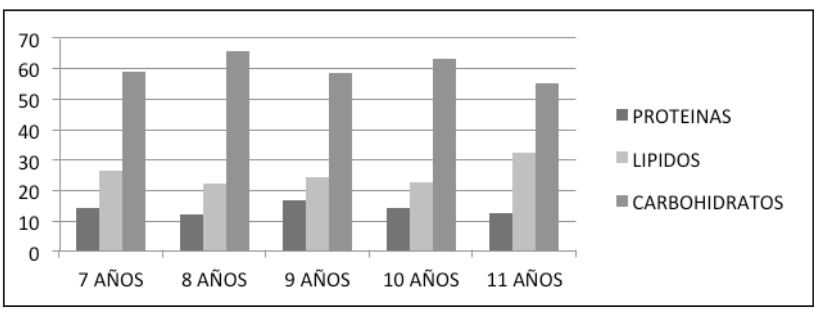

Figura 4. Distribución energética de las dietas de escolares en función a su edad

and Human Services de EE.UU. (2005) y se aprecia en la Figura 4 y la Tabla 4.

Ingesta de hierro y concentración de hemoglobina en escolares

El consumo promedio de hierro entre los escolares evaluados fue de $10,9 \mathrm{mg} /$ día, a nivel nacional se ha registrado una media de consumo de hierro de $7,2 \mathrm{mg} /$ día entre niños de 5 a 14 años, la relación entre ingesta de hierro y niveles de hemoglobina fue de $0,369,(P=0,176)$.

Ingesta de energía en madres

La media de consumo de energía materna fue de $1907,21 \pm 533,5 \mathrm{kcal}$, el mayor aporte de energía de la dieta provino de los carbohidratos $(1167 \pm 391,12 \mathrm{kcal})$, seguido por los lípidos $(499,54 \pm 138,47 \mathrm{kcal})$ y las proteínas $(240,62 \pm 74,4 \mathrm{kcal})$; los carbohidratos aportaron con el $61,19 \%$ de la energía de la dieta, el aporte de lípidos y proteínas fue de $26,19 \%$ y $12,62 \%$, respectivamente, similar a las recomendaciones del Deparment of Health and Human Services de EE.UU. (2005). La distribución energética de la dieta materna se puede apreciar en la Fig. 5.

Se registró una fuerte correlación entre el consumo de energía y el IMC $(\mathrm{r}=0,834 ; \mathrm{p}=0,001)$; esta asociación

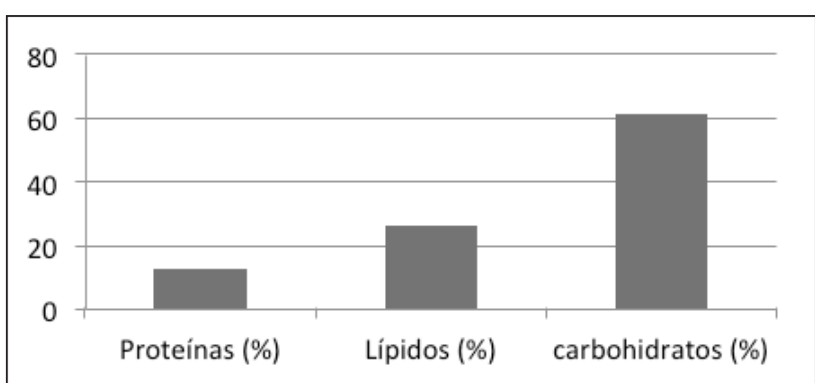

Figura 5. Distribución energética de la dieta de las madres de familia. entre el IMC y la ingesta de energía probablemente se deba a que los requerimientos energéticos de mujeres con sobrepeso son mayores que los de mujeres con peso normal (De Boer et al., 1997). En la presente investigación la energía proveniente de proteínas, carbohidratos y lípidos se asocia positivamente con el incremento del IMC, aunque en el caso de los lípidos dicha asociación no es significativa. El $25 \%$ de las entrevistadas presentaron sobrepeso y el $16,67 \%$, obesidad, no obstante una baja ingesta promedio de energía $(1.907,21 \mathrm{kcal})$, por lo que la prevalencia de sobrepeso y obesidad podría relacionarse con subestimación de ingesta de energía u otros factores como la escasa actividad física (Rojas et al., 2004).

En el presente estudio se observó una alta y positiva correlación entre la ingesta de energía y el perímetro de cintura ( $\mathrm{r}=0,777 ; \mathrm{p}=0,003)$; Halkjær et al. (2006) no hallaron asociación entre estas variables en mujeres, aunque observaron una relación inversa entre el CC y la ingesta de proteínas; la ingesta de carbohidratos refinados y grasa de origen vegetal guarda relación con el incremento de la CC. Aunque se evidencian tendencias, en la presente investigación la ingesta de energía no tuvo una asociación significativa con la proporción de grasa corporal. ( $\mathrm{r}=0,533$; $\mathrm{p}=0,091)$, en contraste, Larson, Tataranni, Ferraro y Ravussin (1995) encontraron en USA, que el porcentaje de grasa corporal se asocia de manera directa con la ingesta de energía y grasa, siendo el porcentaje de energía proveniente de la ingesta de grasa mayor en mujeres obesas que en mujeres delgadas.

La ingesta de hierro en las madres de familia fue de 8,12 $\mathrm{mg} / \mathrm{día}$, mientras que el promedio de hemoglobina fue de $11,88 \mathrm{~g} / \mathrm{dl}$, existiendo un nivel de correlación positivo, aunque bajo y no significativo, entre ambas variables $(\mathrm{r}=0,225, \mathrm{p}=0,561)$. En mujeres peruanas en edad fértil (INS, 2008), la media de ingesta de hierro es $7,4 \mathrm{mg} /$ día y la prevalencia de anemia es de $38,2 \%$, inferior a la observada en el presente estudio. La elevada prevalencia de anemia podría explicarse por una baja ingesta de este metal ya que los requerimientos para mujeres en edad fértil oscilan entre 19,6 a 58,8 mg diarios de hierro, en función de su biodisponibilidad. En el caso de mujeres en edad fértil la menstruación y la gestación son factores predisponentes para sufrir deficiencia de hierro o anemia (Frewin et al., 1997), y podrían explicar la escasa relación hallada en el presente estudio entre la ingesta de hierro y los niveles de hemoglobina.

\section{Conclusiones}

El exceso de peso (sobrepeso y obesidad) y anemia son los principales problemas nutricionales en escolares y sus madres, se observa baja prevalencia del retraso en el crecimiento, esto determina que la prevalencia de coexistencia de sobrepeso materno y retraso en el crecimiento sea baja, 3,19\%, mientras que el 20,21\% presentó coexistencia de exceso de peso en escolares y sus madres; asimismo, en el 21,67\% se observó coexistencia de anemia materna y en escolares, estos problemas nutricionales se presentan pese al bajo consumo de energía, 
la anemia se puede atribuir a la escasa ingesta de hierro. Por otra parte, la circunferencia de cintura se relacionó de manera estrecha con el IMC y porcentaje de grasa corporal.

\section{Agradecimientos}

Se agradece a la Lic. María Camahuali Sánchez, Sub directora del Colegio № 1140 "Aurelio Miró Quesada", por la apertura brindada a la presente investigación; de igual manera se reconoce el aporte de la Srta. Valeria Castillo por su participación en la ejecución de este trabajo.

\section{Literatura citada}

Agudelo, G.; Cardona, O.; Posada, M.; Montoya, M.; Ocampo, N.; Marín, C.; Correa y López, C. 2003. Prevalencia de anemia ferropénica en escolares y adolescentes. Rev. Pamam. Salud Pública, 13 (6): 376386.

American Medical Association. 2007. Assessment and Management of Adult Obesity: A Primer for Physicians. Recuperado de http://www.ama-assn.org/ama/pub/ category $/ 1093 / \mathrm{html}$

Asociación Benéfica PRISMA. 1996. Laminario de Medidas Caseras. Programa de Alimentación y Nutrición de la Familia en Alto Riesgo. Lima, Perú: A. B. Prisma.

CEPAL/FAO/PMA. Hambre y cohesión social. Cómo revertir la relación entre inequidad y desnutrición en América Latina y el Caribe. Recuperado de http:// http:// goo.gl/JU24b9

Collazos, C. 2004. Tablas Peruanas de Composición de los Alimentos. (7ma. edición). Lima, Perú: Ministerio de Salud. Instituto Nacional de Salud. Centro Nacional de Alimentación y Nutrición. Editorial Acuario S.A.

De Boer, J.; Van Es, A.; Van Raaij y Havtavast, J . 1997. Energy requirements and energy expenditure of lean and overweight women, measured by indirect calorimetry. $\mathrm{Am}$ J Clin Nutr, (46), 13-21.

EC-FAO. 2007. Food security information for action program. Distance learning to support capacity building and training for national and local Food Security information systems and networks. Nutritional Status assessment and analysis. Lesson 3. Nutritional status indicators.

Farias et al. 2007. Comparison of the anthropometric and biochemical variables between children and their parents. ALAN, Vol. 57(2).

Frewin, R.; Henson, A. y Provan, D. 1997. ABC of Clinical haematology: Iron deficiency anaemia. $B M J$, 314(7077), 360.

Grillo, L.; Carvalho, L.; Silva, A. y Sawaya, A. 2000. Influence of socio-economic conditions in the nutrition changes and a rest metabolic rate in school children living in shantytown of the city of Sao Paulo, Brasil. Rev. Assoc. Med. Bras., 46 (1): 7-14.

Gross, Rainer, Lechtig, Aarón y López de Romaña Daniel. 2006. Baseline evaluation of nutritional status and government feeding programs in Chiclayo, Peru. Food and Nutrition Bulletin, 27(4): S115-S121.

Halkjær, J.; Tjønneland, A.; Thomsen, B.; Overvad, K. y Sørensen; T. 2006. Intake of macronutrients as predictors of 5-y changes in waist circumference. Am J Clin Nutr., 84, $789-797$.

Hassan, K.; Sullivan, K.; Yip, R. y Woodruff, B. 1997. Factors Associated with Anaemia in Refugee Children. $J$. Nutr., 127: 2194-2198.

Hirschler, V.; Delfino, A.; Clemente, G.; Aranda, C.; Calcagno, M.; Pettinicchio, H. y Jadzinsky, M. 2005. ¿Es la circunferencia de cintura un componente del síndrome metabólico en la infancia? Arch. argent. Pediatric, 103(1): 1-7.

Hoffman, D.; Sawaya, A.; Verreschi, I.; Tucker, K. y Roberts, S. 2000. Why are nutritionally stunted children at increases risk of obesity? Studies of metabolic rate and fat oxidation in shantytown children from Sao Paulo, Brasil. Am J Clin Nutr., 72(3): 702-707.

Instituto Nacional de Salud (INS). (s/f). Situación Nutricional del Perú. Disponible en http://goo.gl/1Bm9dx Larson, D.; Tataranni, P.; Ferraro, R. y Ravussin, E. 1995. Ad libitum food intake on a cafeteria diet in nature American women: relations with body composition and 24 h energy expenditure. Am J Clin Nutr., 62(5): 911-917.

Lechtig, A.; Cornale, G.; Ugaz, ME. y Arias, L. 2009. Decreasing stunting, anaemia, and vitamin A deficiency in Peru: results of the Good Start in Life Program. Food and Nutrition Bulletin, 30(1): 37-48.

Llanos, F. y Cabello, E. 2003. Distribución del índice de masa corporal (IMC) y prevalencia de obesidad primaria en niños pre-púberes de 6 a 10 años de edad en el distrito de San Martín de Porres - Lima. Rev. Med. Hered., 14: 107-110.

Miespireta, M.; Rosas, A.; Velásquez, J.; Lescano, A. y Lanata, C. 2007. Transición nutricional en el Perú, 19912005. Rev. Perú Med. Exp. Salud Pública, 24(2): 129-135. Ministerio de Educación/UNICEF/FOCONDES/PMA. 1993. I Censo Nacional de talla en escolares. Lima, Perú. National Center for Health Statistics. (2000). GrowCharts: United States. Hyattsville, MD. Recuperado de http:// www.cdc.gov/growtcharts

Pajuelo, J.; Muñoz, C.; Ayquipa, A.; Ponciano, W. y López, R. (s/f). El Sobrepeso, la Obesidad y la Anemia Nutricional en la Mujer Adulta 2000. Recuperado de http://goo.gl/cbP1Hu

Pajuelo, J.; Rocca, J. y Gamarra, M. 2003. Obesidad infantil: sus características antropométricas y bioquímicas. Anales de la Facultad de Medicina.

Pajuelo, J.; Vergara, J. y Cruz, G. 2001. Coexistencia de problemas nutricionales en niños de 6 a 9 años de edad, de centro educativos estatales de Matucana, Santa Eulalia y Lima. Anales de la Facultad de Medicina, 62 (4). Universidad Nacional Mayor de San Marcos.

Perez, E.; Hendricks, M.; Beard, J.; Murray-Kolb, L.; Berg, A.; Tomlinson, M.; Irlam, J.; Isaacs, W.; Njengele, T.; Sive, A. y Vernon-Feagans, L. 2005. Mother-Infant Interactions and Infant Development Are Altered by Maternal Iron Deficiency. Anemia J. Nutr., 135: 850-855. Piazza, N. 2005. La circunferencia de cintura en los niños y adolescentes. Arch. argent. pediatr., 103(1), 5-6.

Queiroz, C.; Ribeiro, R.; Oliveira, A.; Pinto, E.; 
Fiaccone, R. y Pinheir, S. 2009. Fatores associados à anemia em crianças e adolescents de escolas públicas de Salvador, Bahia, Brasil. Cad Saude Pública, 25(4): 877888.

Quizphe, ES.; Hurtig, A. y Llamas, A. 2003. Prevalencia de anemia en escolares de la zona amazónica de Ecuador. Rev. Panam. Salud Pública, 13(6): 355-361.

Rojas, C.; Moreno, C.; Vara, E.; Bernui, I. e Ysla, M. 2004. Consumo de energía y nutrientes, características socioeconómicas, pobreza y área de residencia de mujeres peruanas en edad fértil. Revista peruana de medicina experimental y salud pública, 21(4): 231-239.

Sawaya, L. y Roberts, S. 2003. Stunting and future risk of obesity: principal physiological mechanisms. Cad Saude Pública, 19 (Suppl. 1).

Stoltzfus, R.; Chwaya, H.; Tielsch, J.; Schulze, K.; Albonico, M. y Savioli, L. 1997. Epidemiology of iron deficiency anaemia in Zanzibari schoolchildren: the importance of hookworms. Am J Clin Nutr, 65, 153-159.

Torres, H.; Caballero, J.; Ponce, J.; Valverde, I. 2004. Evaluación nutricional de niños entre 6 y 9 años de dos colegios de la costa y la sierra. En I Congreso Internacional VII Congreso peruano de nutrición "Alimentación y Nutrición para una vida sustentable" 2004, Lima.

Wait, B.; Blair, R. y Roberts, L. 1969. Energy intake of well-nourished children and adolescents. Am J Clin Nur, (22), 1383-1396.

U.S. Department of Health and Human Services and U.S. Department of Agriculture. 2005. Dietary Guidelines for Americans. Recuperado de http//: www. healthierus.gov/dietaryguidelines

World Health Organization [WHO]. 2001. Iron Deficiency Anaemia Assessment, Prevention and Control. A guide for programme managers. Recuperado de http:// http://goo.gl/kdII4l 